\title{
Inhibition of angiotensin II type I pathway reduced tumor growth and ameliorates fibrosis/inflammation associated with colorectal cancer
}

Fereshteh Asgharzadeh",2, *, Asma Mostafapour ${ }^{3}$, Forouzan Amerizadeh2,3,4, Amir Avan ${ }^{2,3,4,}$, Farzad Rahmani ${ }^{5}$, Reihaneh Sabbaghzadeh ${ }^{6}$, Seyed Mahdi Hassanian ${ }^{3,7}$, Maryam Fakhraei ${ }^{1}$, Alieh

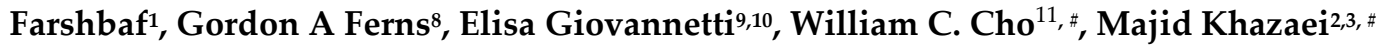

1) Department of Medical Physiology, Faculty of Medicine, Mashhad University of Medical Sciences, Mashhad, Iran.

2) Student Research Committee, Faculty of Medicine, Mashhad University of Medical Sciences, Mashhad, Iran.

3) Metabolic syndrome Research center, Mashhad University of Medical Sciences, Mashhad, Iran

4) Department of Medical Genetics, Faculty of Medicine, Mashhad University of Medical Sciences, Mashhad, Iran.

5) Cancer Research center, Mashhad University of Medical Sciences, Mashhad, Iran

6) Department of Biology, Faculty of Science, Hakim Sabzevari University, Sabzevar, 96179-76487, Iran

7) Department of Medical Biochemistry, Faculty of Medicine, Mashhad University of Medical Sciences, Mashhad, Iran.

8) Brighton \& Sussex Medical School, Division of Medical Education, Falmer, Brighton, Sussex BN1 9PH, UK.

9) Cancer Pharmacology Lab, Fondazione Pisana per la Scienza, Pisa, Italy;

10) Department of Medical Oncology, Cancer Center Amsterdam, VU University Medical Center, Amsterdam, The Netherlands

11) Department of Clinical Oncology, Queen Elizabeth Hospital, Kowloon, Hong Kong, China

\# Corresponding Authors:

Majid Khazaei MD PhD, and Metabolic Syndrome Research center, Mashhad University of Medical Sciences, Mashhad, Iran Tell: +98 513 8002298; E-mail: Khazaeim@mums.ac.ir

William C. Cho,Ph.D. Department of Clinical Oncology, Queen Elizabeth Hospital, Kowloon, Hong Kong, China williamcscho@gmail.com

* Fereshteh Asgharzadeh and Amir Avan, equally contributed as first author

Abstract: Dysregulation of the angiotensin-II Type-I receptor (AT1R) and its pathway was reported to associate with poor-prognosis in several malignancies, including colorectal-cancer (CRC). We have explored the therapeutic-potential of targeting AT1R using valsartan, and its pharmacologicalinteraction with Fluorouracil (5-FU) in CRC. Anti-proliferative function was evaluated in 2-/3dimensional cells and in vivo models. Anti-proliferative, anti-migratory, apoptotic function and effect on cell-cycle was assessed by 3-(4,5-dimethylthiazol-2-yl)-2,5-diphenyltetrazolium bromide (MTT), wound-healing test, and Fluorescence-activated cell sorting (FACS), respectively, while gene-expression was determined at mRNA/protein levels. By histogical analysis and measuring of oxidative/antioxidant markers, we evaluated the anti-inflammatory properties of valsartan. Valsartan suppressed cell-growth and impacted the anti-tumor-activities of 5-FU by apoptosisinduction. Valsartan inhibited the cells migration by perturbation of Matrix metalloproteinase 
(MMP1). Furthermore, valsartan inhibited tumor-growth and metastasis, and this was more notable in valsartan/5-FU combination-treated-group. The mechanism was plausible to be via the induction of Reactive-oxygen-species (ROS) and down-regulation of Superoxide-dismutase (SOD), thiol/catalase (CAT) as well as Vascular endothelial growth factor (VEGF) and Transforming growth factor beta (TGF- $\beta$ ). Valsartan may protect cells against intestinal fibrosis by modulation of profibrotic and pro-inflammatory components include fibronectin, Interleukin) IL-1 $\beta$ (, Tumor necrosis factor alpha) TNF- $\alpha$ (, Interferon gamma) INF- $\gamma$ (, and Monocyte Chemotactic Protein 1 (MCP-1). Our findings demonstrated that targeting the AT1R receptor may inhibit tumor-growth and ameliorate fibrosis and inflammation associated with CRC via modulation of AT1 and TGF- $\beta$ pathways.

Keywords: Angiotensin-II Type-I receptor; renin-angiotensin system; valsartan; colorectal cancer

\section{Introduction}

Colorectal cancer (CRC) is a common cancer for death globally, and some cases develop lungs metastasis [1]. The renin-angiotensin system (RAS) has an major role in blood pressure regulation and fluid body homeostasis [2]. However, there is increasing evidence that RAS may also influence cellular apoptosis, proliferation, cell adhesion and inflammation [3]. Moreover angiotensin II (AT II), was proposed to improve cancer cell development and metastasis [4]. RAS pathway activity depends on the action of ATII, which is related to Angiotensin-I-converting enzyme (ACE), the action of which AT1R antagonists and ACE inhibitors can be blocked. Renin-angiotensin system inhibitors (RASIs) are well known for their potential function in suppressing tumor progression in CRC [5], and there are suggestions that they play a significant role in tumor cell lines inhibiting growth and angiogenesis $[6,7]$. Although several epidemiological reports have reported that RASIs suppress the growth and metastasis of various cancers [4]. It has been shown in the model of gastric cancer that valsartan can inhibit the tumor growth and suppress the tumor angiogenesis [8]. In addition, Wang study was designed to identify the effects of valsartan with $\gamma$-rays on the expression of vascular endothelial growth factor (VEGF), radiation sensitivity, invasive potential and proliferative activity of nasopharyngeal carcinoma (CNE-2) in vitro. Wang's research showed that valsartan prevented the proliferation and invasion by CNE-2 cells of the nasopharyngeal carcinoma line and increased its radiation sensitivity [9]. Clinical studies have shown that use of valsartan compared to other ARBs in patients with hypertension significantly decreases inflammatory markers [10-12] .In addition, the results of the meta-analysis showed an increase in neoplastic diseases using candesartan, losartan and telemizartan, which was not significant, while the incidence of neoplastic diseases using valsartan showed a significant decrease [13] .The potential of RASIs as chemopreventive agents against CRC is a subject of interest. To evaluate this association, we have investigated the therapeutic potential and the molecular mechanisms of actions of valsartan (one of the family members of the AT1R antagonist) in CRC progression and metastasis

\section{Results}

\subsection{Inhibition of cell proliferation, migration and induction of apoptosis}

To evaluate the anti-cancer results of valsartan and valsartan + 5-FU, the viability of CT26 cells was tested using MTT assay. As shown in Fig. 1A, valsartan, and 5-FU dose-dependently inhibited cell growth. Results showed that co-treatment of valsartan and 5-FU decreased the IC50 value of 5-FU (IC50 valsartan $=1 \mathrm{mM}$ and IC50 5-FU=10 $\mu \mathrm{M}$ ). To assess the potential inhibitory effect of valsartan on the migration of CRC cells, the effect of valsartan on the expression of matrix metalloproteases-1 (MMP-1) was evaluated. The potential migratory behavior of valsartan-treated CRC cells was significantly inhibited as comparison with the untreated group $(\mathrm{P}<0.05)$ (Fig. 1B, C). As well as, the administration of valsartan decreased expression the enzymatic activities of MMP-1 as visualized by 
qPCR (Fig. $1 \mathrm{D}$ ). Also we found that valsartan increased in both early (annexin V positive/ PI negative) and late (annexin $\mathrm{V}$ and PI positive) apoptosis (Fig. 1E, F).

A

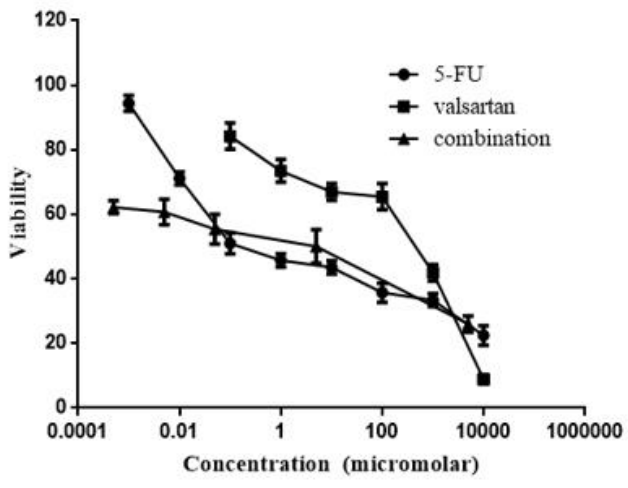

B

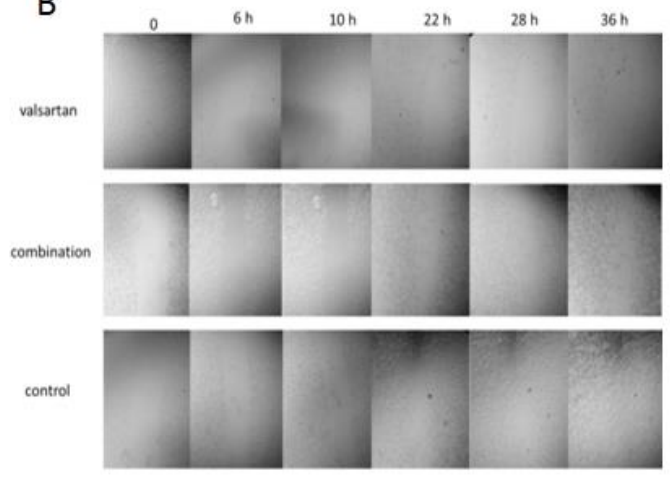

$\mathrm{F}_{100}$
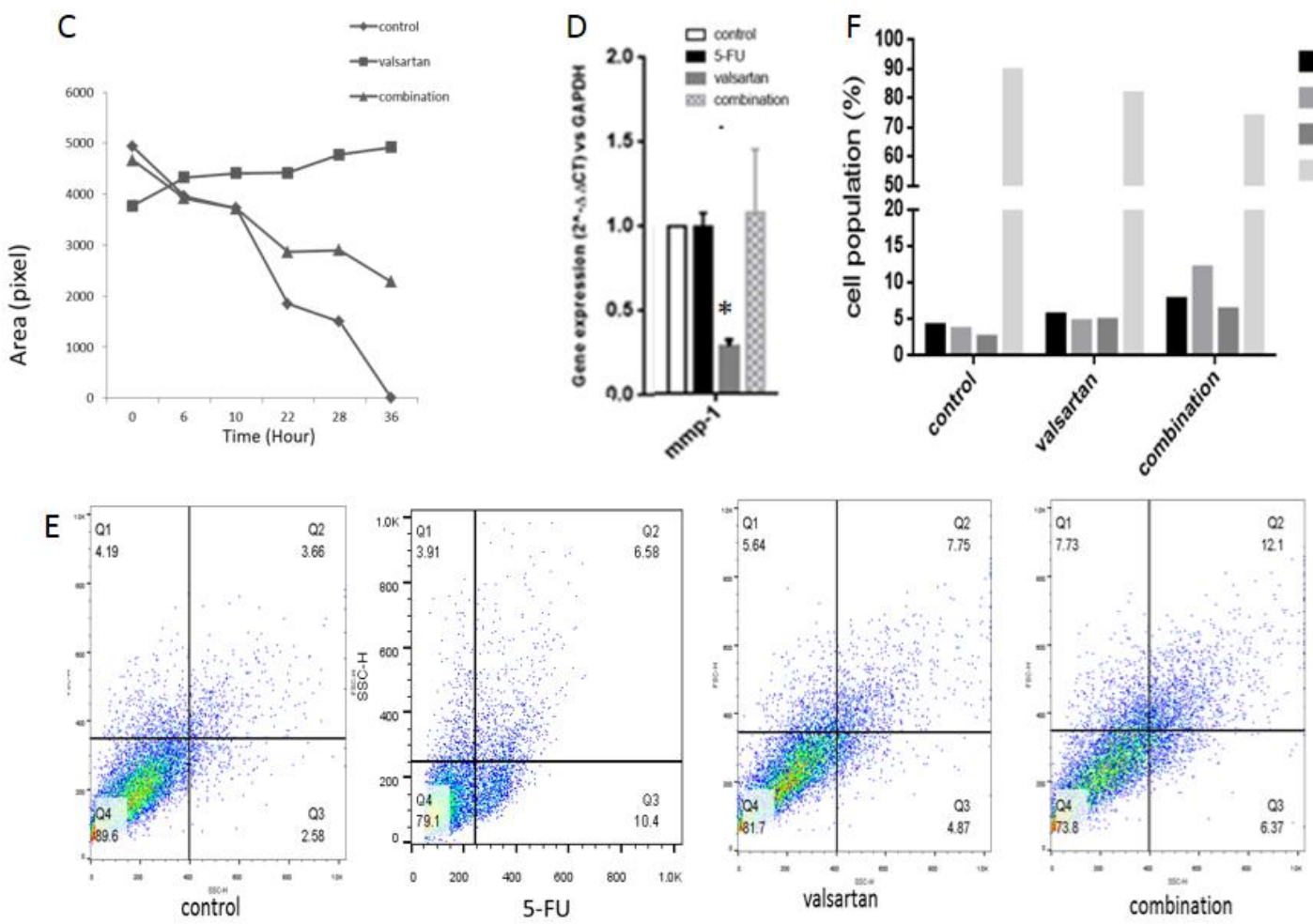

Figure 1. Valsartan inhibits cell migration and induces apoptosis of CRC cells. (A) Growth inhibitory effects of valsartan (1mM) after 24,48 and $72 \mathrm{hr}$ exposure CT-26 cells. (B, C) Effect of valsartan on the migration of the CT-26 cells. (D) Expression level MMP-1 in CRC cells treated with valsartan as detected by real-time RT-PCR. (E, F) CT-26 cells were treated with valsartan for $24 \mathrm{~h}$ and apoptosis was explored by flow cytometry using annexin V/PI staining. The values of the lower right and the upper right area indicate the percentage of the cells in early and late apoptosis, respectively.

\subsection{Inhibition of tumor growth and lung metastatic}

Our data showed that the administration of valsartan reduced tumor size compared to control and 5-FU group (Fig. 2A-B). Fig 2B shows a reduction in the size of tumor significantly between combination and 5-FU group. Histopathological changes of the tumor are shown in the sections stained with H\&E are shown in Fig. 2C. Administration of valsartan and 5-FU enhance tumor necrosis and tumor density but in valsartan-treated group tumor necrosis and tumor density higher than that 5-FU group as the standard chemotherapeutic regiment in CRC (Fig. 2C). Administration of valsartan decreased tumor density compared to the control group. Using Trichrome stain shows 
that treatment with valsartan decreased vascular density (Fig. 2D), fibrotic tissue and collagen deposition in tumor tissue compared to the control group. Additionally, valsartan in combination with 5-FU decreased the expression level of VEGF, IL-1, MCP-1, fibronectin, collagen type1 genes (Fig. 2E). Interestingly, our finding illustrated that valsartan was able to inhibit lung metastasis and improve body weight (Figure 3A-H), compared to the control and 5-Fu group. Also this effect was more pronounce in the mice which were received valsartan plus 5-FU (Fig. 3) in both macroscopic and microscopic tumor nodules examinations (Fig 3).

A

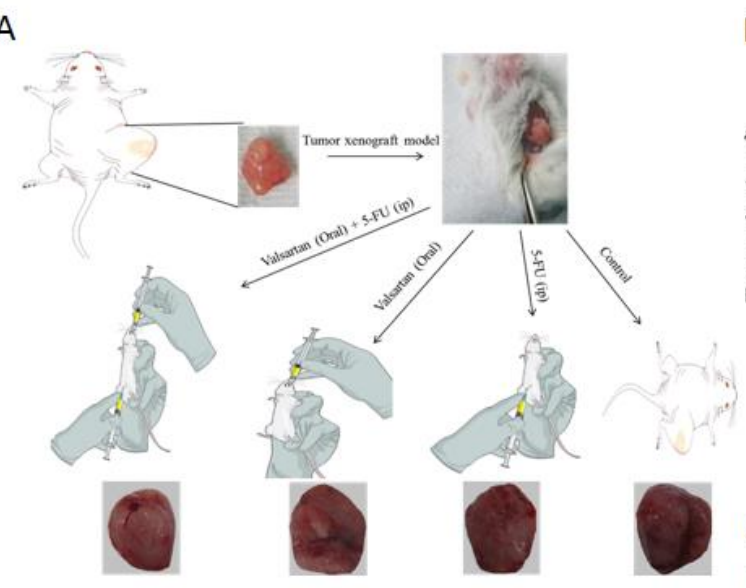

D
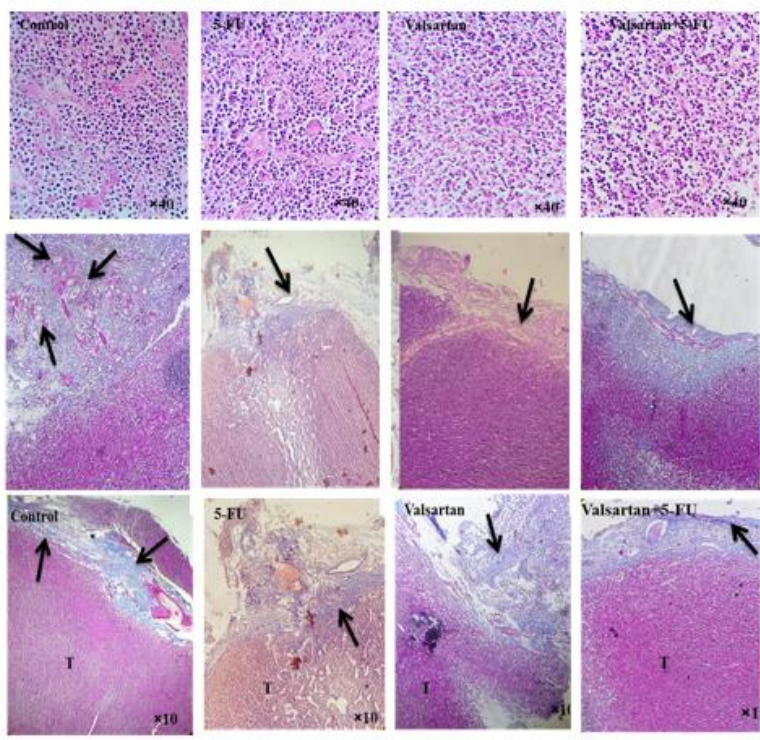
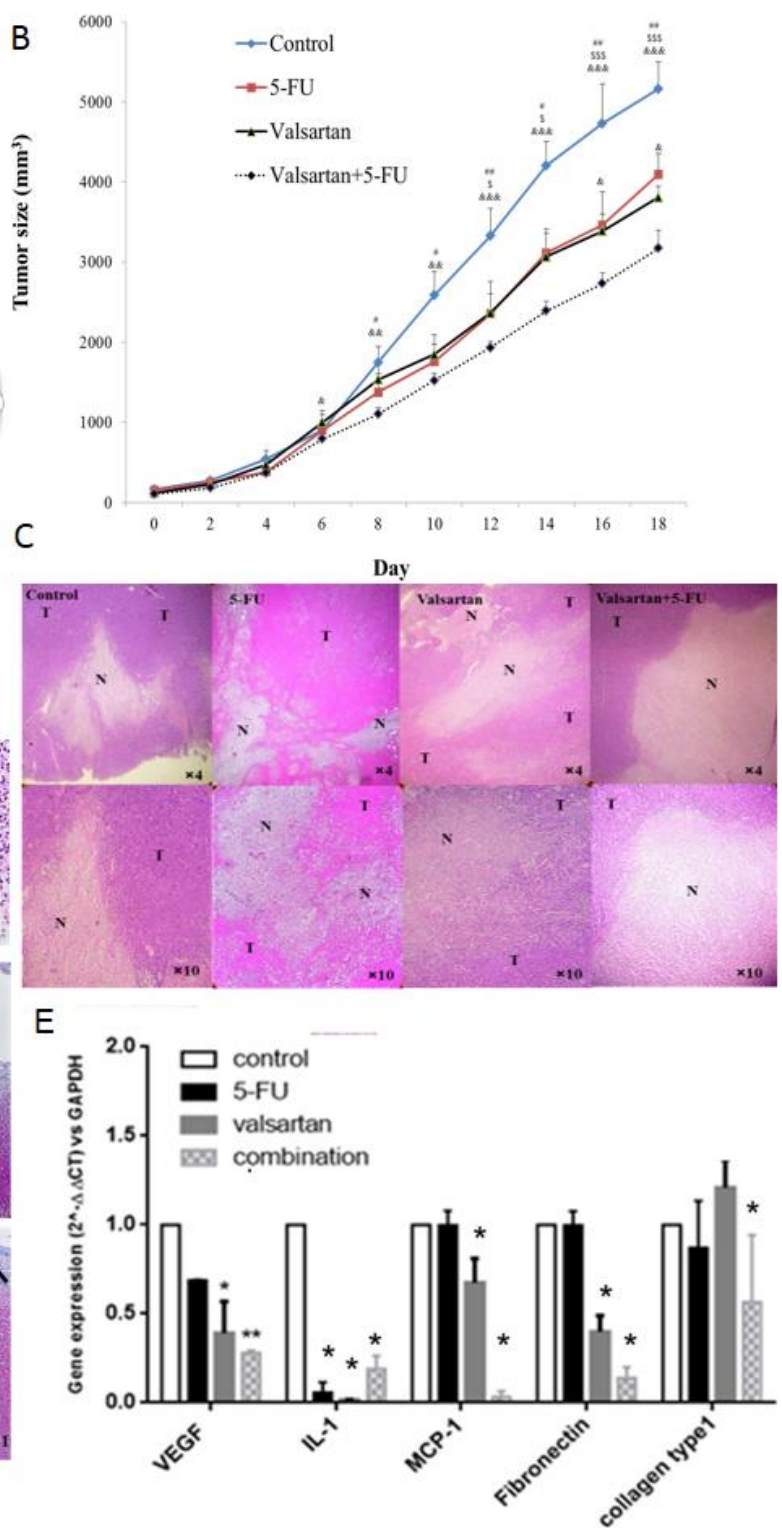

Figure 2. Valsartan suppresses tumor growth. (A) The treatment schedules and development of colorectal cancer models. Schematic of the Mice-derived tumor xenograft model. Tumor tissues were retranslated into Balb/c mice $(n=24)$. After the tumor volume reached to $80-100 \mathrm{~mm} 3$, the treatment was initiated for 18 days. Relative variations of tumor volumes in different groups. (B) Tumor volumes were monitored every other day using a digital caliper. Representative H\&E staining showed that tumor necrosis indicated by the $\mathrm{N}$ (necrosis) and $\mathrm{T}$ (tumor) in each group. magnification was mention under each figure. (C) Representative H\&E staining showed that Tumor density and (D) images of Masson's trichrome staining were showed that Vascular density and Tumor fibrosis in each group. magnification was mention under each figure. (E) The expression level of VEGF, IL-1, MCP-1, fibronectin, collagen type1 genes in CT26 cells treated with valsartan as detected by real-time RT-PCR. 


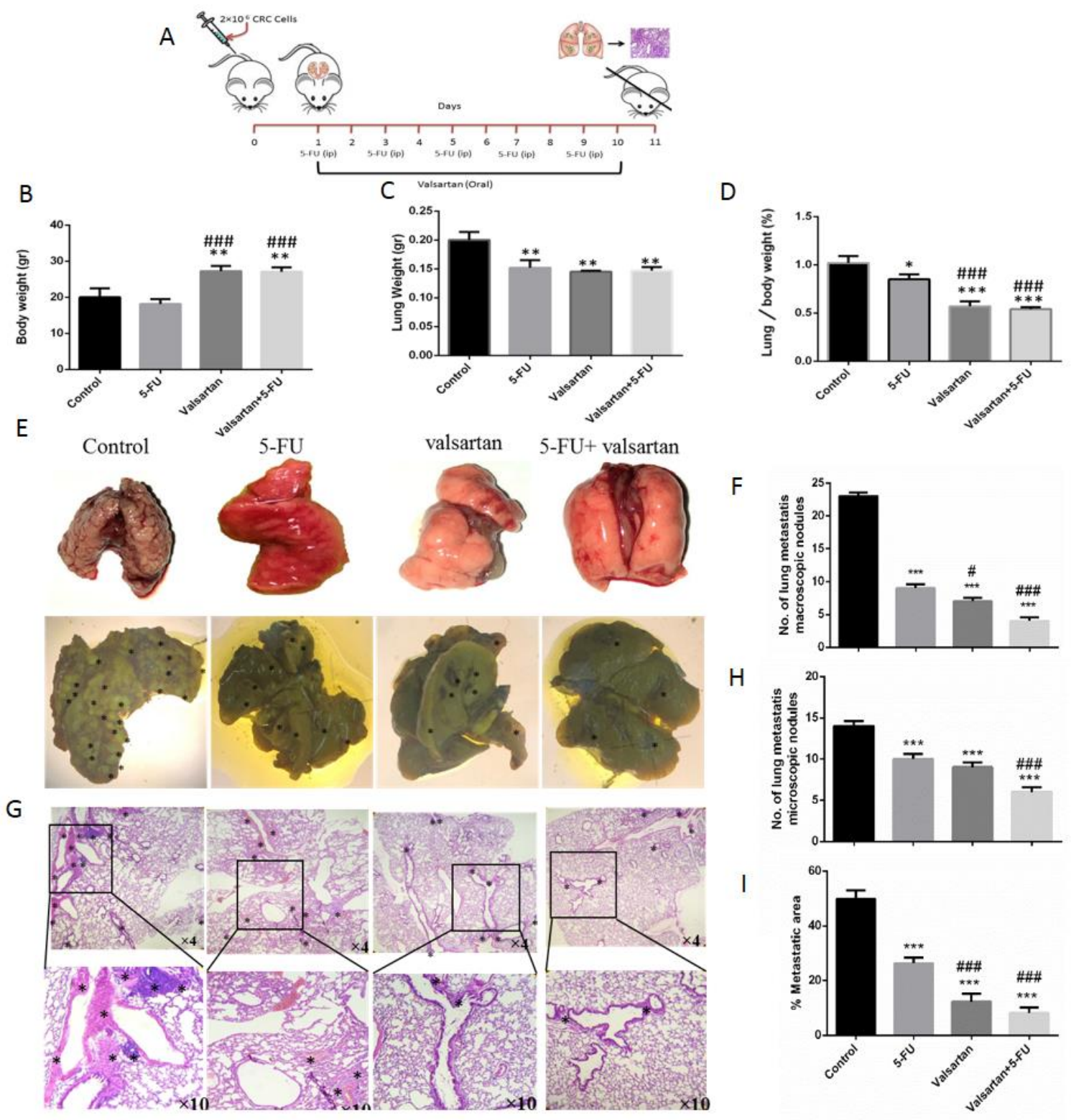

Figure 3: Effect of valsartan on the lung metastatic. (A)The treatment schedules and schematic representation of experimental protocol of lung metastasis derived CT-26 model. Twenty-four male inbred BALB / c mice were received $2 \times 10^{6} \mathrm{CT} 26$ cells by tail intravenous injection and treated 24 hours after the cell injection for 10 days. Control group received normal saline. 5-FU group received $5 \mathrm{mg} / \mathrm{kg} /$ every other day; intraperitoneal. Valsartan group received $40 \mathrm{mg} / \mathrm{kg} /$ day; oral gavage. Combination group received 5 -FU ( $5 \mathrm{mg} / \mathrm{kg} / \mathrm{every}$ other day; ip) plus (valsartan $40 \mathrm{mg} / \mathrm{kg} / \mathrm{day}$; oral gavage); (B)The analysis showed that valsartan treatment significantly increased body weight (C) and decreased lung weight (D) and the lung/body weight ratio, compared with the control group $\left({ }^{*} \mathrm{P}<0.05,{ }^{* *} \mathrm{P}<0.01\right.$ and ${ }^{* * *} \mathrm{P}<0.001$ compare with control group and \#\#\#P<0.001 compare with 5-FU group). (E, F) Observational analysis showed that valsartan administration decreased the number of macroscopic metastatic lung nodules. Furthermore, the evaluation of tumor formation with Bouin's solution showed that valsartan treatment significantly reduced the number of macroscopic metastatic lung nodules. (G) HE staining demonstrated valsartan treated groups decreased higher number of (H) microfoci and (I) percent of metastatic area in lung significantly (The asterisk represent lung microfoci). Magnification was mentioned under each figure $(G)$.

\subsection{Valsartan induced oxidative stress}


The valsartan and combination groups had a higher MDA level compare to the control group $(\mathrm{P}<0.001$ for both). A significant increase in MDA concentration was observed in the valsartan and valsartan-5-FU group compare to the 5 -FU group ( $\mathrm{P}<0.01$ and $\mathrm{P}<0.001$ respectively) (Fig. $4 \mathrm{~A}$ ). Administration of the valsartan decreased the total thiol, CAT and SOD (Fig4B-D). The NO metabolites of tumor tissues of valsartan-treated group and valsartan and combination groups were higher than the control group $(\mathrm{P}<0.05$, Fig. $4 \mathrm{E})$. Moreover, our data showed that valsartan increased ROS generation in CRC cells, as detected by measured by DCFH-DA staining (Fig. 4G-H). These results clearly suggest that valsartan disrupts the antioxidant/oxidant balance, leading to more ROS production.
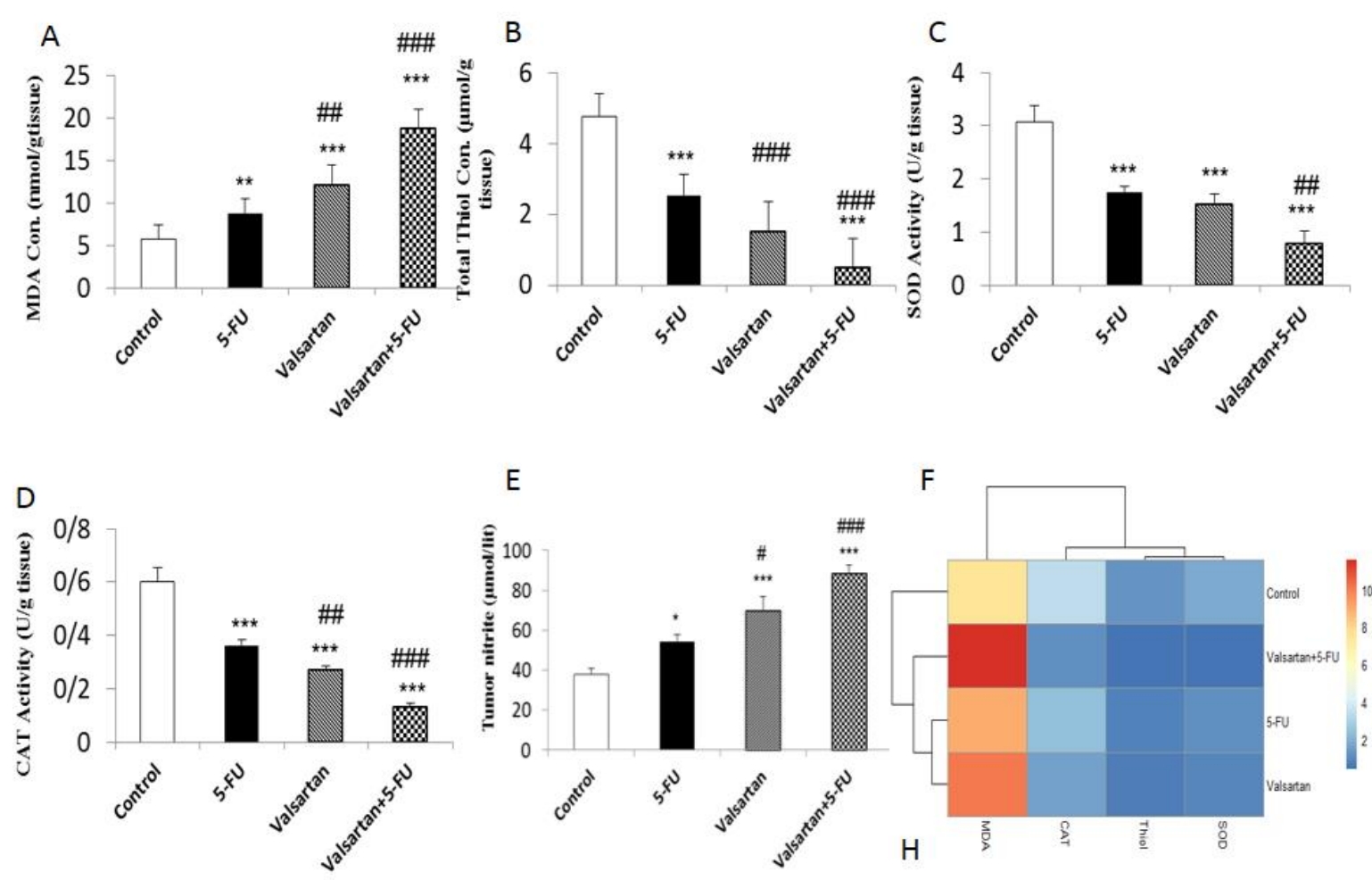

G

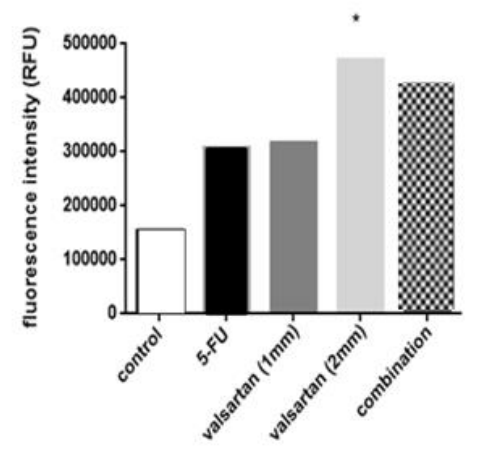

Figure 4: Valsartan induces cell senescence by expression oxidative stress in homogenized colon samples (A) The tumoral MDA concentrations, (B) total thiol content, (C) SOD, (D) CAT and (E) tumoral NO metabolites, (F) Heat map of oxidative stress markers, (G, F) ROS generation. Data are shown as Mean \pm SEM ( $\mathrm{n}=6$ in each group). ${ }^{*} \mathrm{P}<0.05,{ }^{* *} \mathrm{P}<0.01$ and ${ }^{* * *} \mathrm{P}<0.001$ compared to control group. \# P $<0.05$, \# $\mathrm{P}<0.01$ and \# \# $\mathrm{P}<0.001$ compared to 5-FU group. Data are shown as Mean \pm SEM ( $n=6$ in each group). (F) Drug response for MDA concentrations, total thiol content, SOD, CAT. (G) Qualitative characterization of ROS generation using fluorescence microscopy. The stimulatory effect of valsartan on production of cellular ROS was investigated in CT-26 cells treated with two concentrations ( 1 and $2 \mathrm{mM}$ ) of valsartan.

\subsection{The interaction of valsartan with $A T 1 R, T G F \beta$, and inflammatory/anti-inflammatory cytokines}


We first performed in silico and binding energy analyses for valuation of the interaction of valsartan with AT1R, IFN $\gamma$, TGF $\beta$, TNF $\alpha$, MCP-1 and IL-1 $\beta$ (Fig5A-F). Analysis of the amount of hydrogen and hydrophobic bonds between inhibitors and enzymes reveals that the complex has many intermolecular hydrogen bonds, suggesting a higher affinity with AT1R. Our study suggested that the drug interaction would be the better on linkage to hydrogen if functional groups had been having ability in the most interactions. The lowest energy obtained in docking simulations AT1R, IFN- $\gamma$, TGF $\beta, T N F \alpha, M C P-1$ and Il $\beta$ respectively. Based on these observations, angiotensin 2 type 1 receptor is the most potent ligand among the other ligands which binds with valsartan and shows the best binding affinity with AT1R protein structure. Among the six different structures of AT1R, IFN- $\gamma$, TGF $\beta$, TNF $\alpha$, MCP- 1 and Il $\beta$ is found that valsartan with binding affinity -11.5 shows the best docking result. The structure attained from the docking results is shown in Fig. 5. Together, the heat map of the data show in the batch cluster indicates oxidative stress markers and IL-6, VEGF and VEGFR-1 that the clustering algorithm discerned batch to batch variation as the most important source of variation within this data set (Fig. 5). We then evaluated the effect of valsartan on inflammatory cytokines expression at protein level. We found that valsartan administration decreased the expression levels of INF- $\gamma$, TGF- $\beta$, IL-6 and TNF- $\alpha$ in CRC significantly (Fig. 6A-D). Also the expression of VEGF and VEGFR-1 in valsartan or valsartan plus 5-FU group was reduced compared to the control or 5-FU groups (Fig. 6E-G).
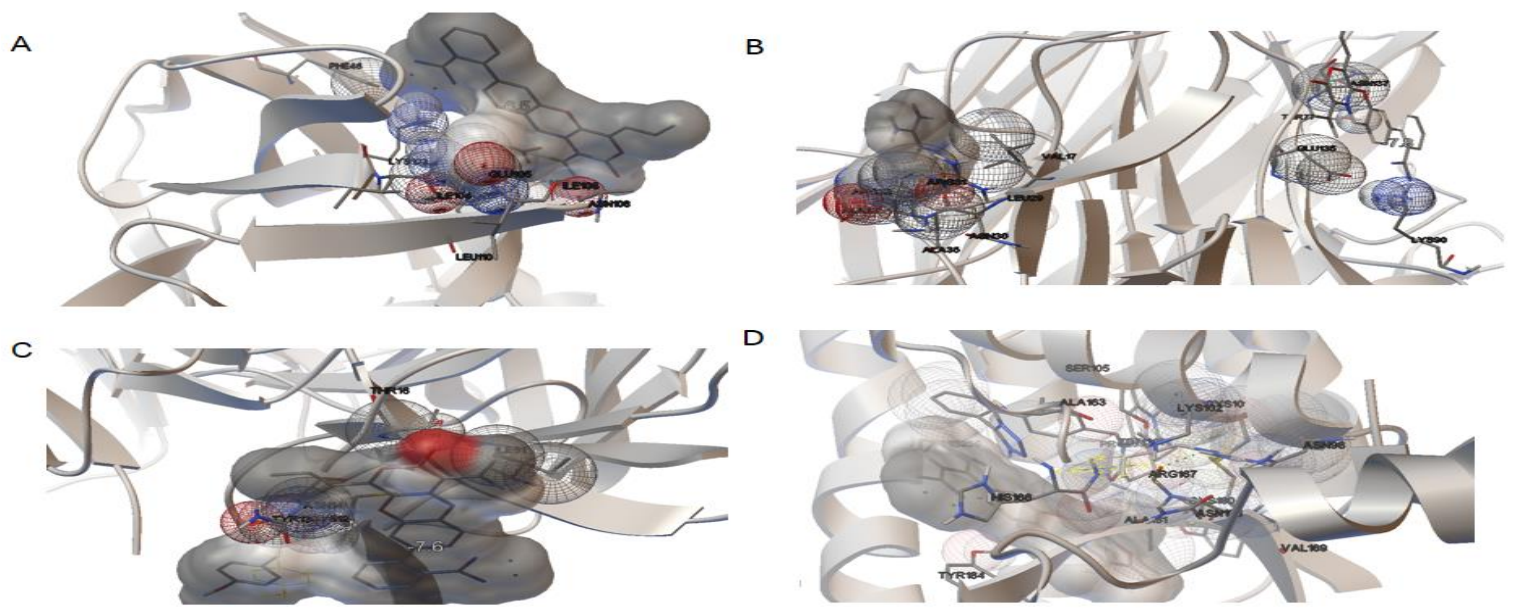

E
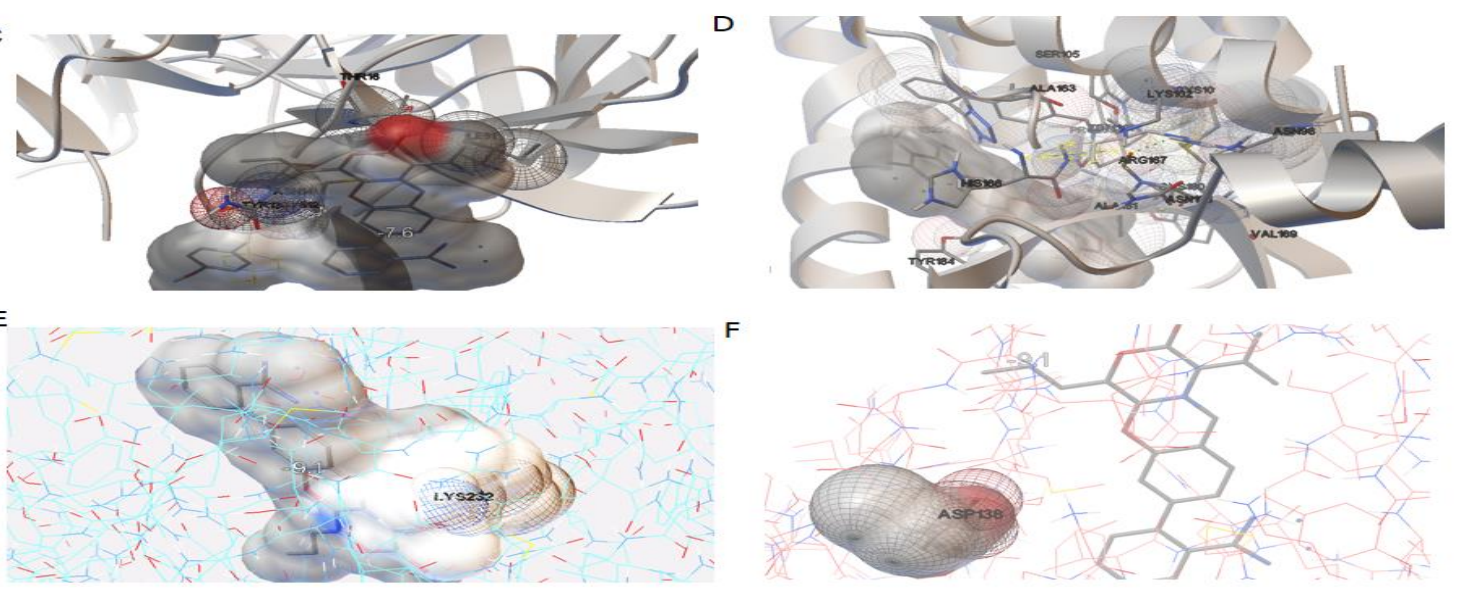

$F$

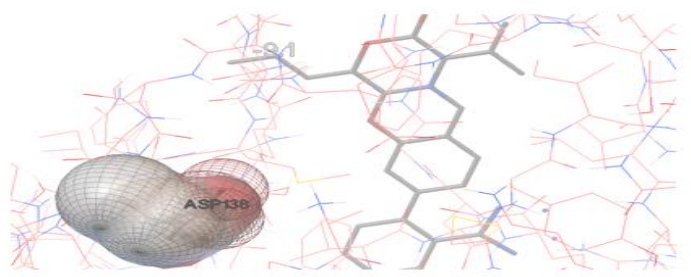

Figure 5: In silico analysis of valsartan response signature. The plots generated by autodock and LigPlot+ software shows interaction between Valsartan and IL- $\beta$ (A), TNF $\alpha$ (B), MCP-1 (C), AT1R (D), TGF- $\beta$ (E). 
A

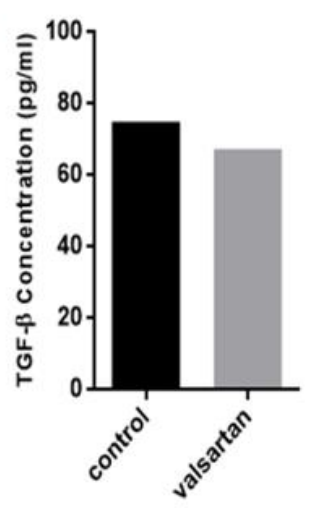

B

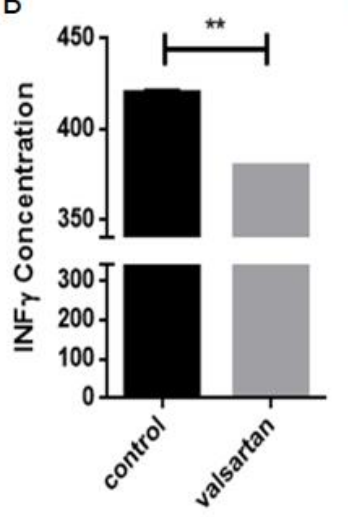

C

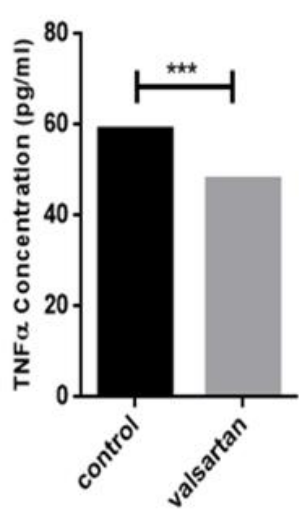

D

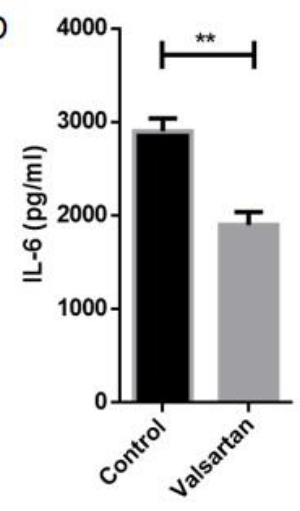

E

F
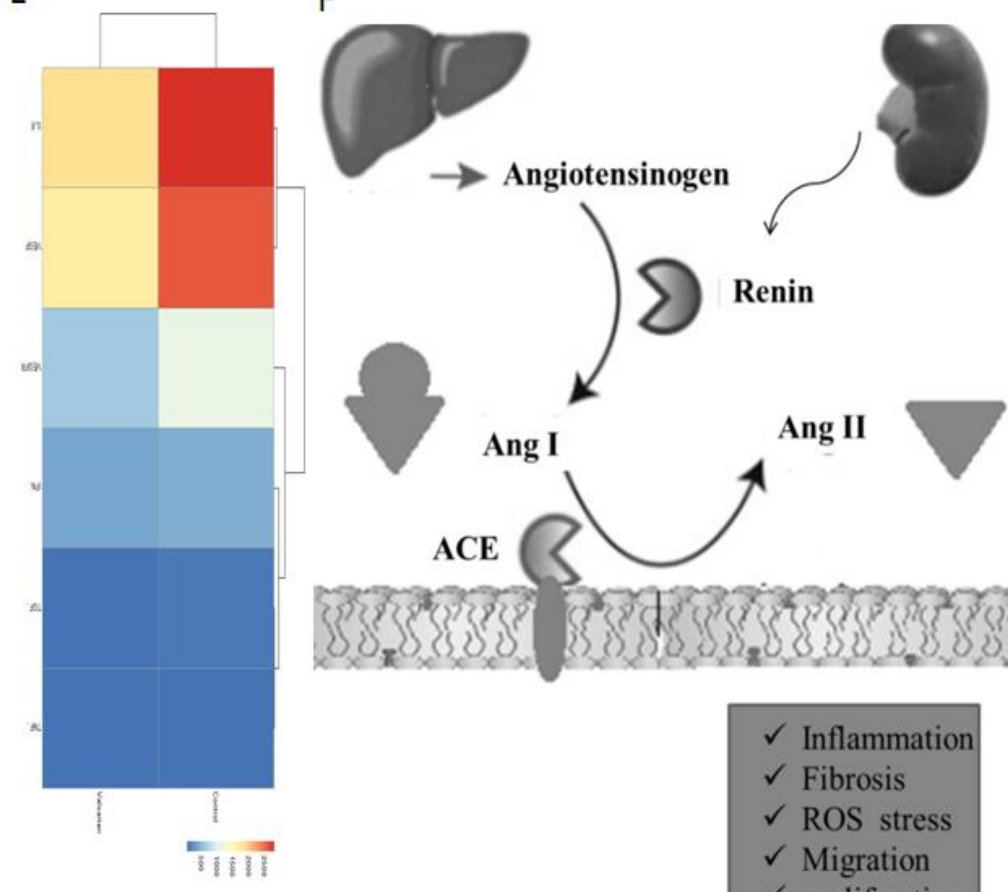

Valsartan

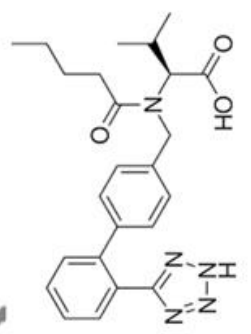

ACE

Ang I

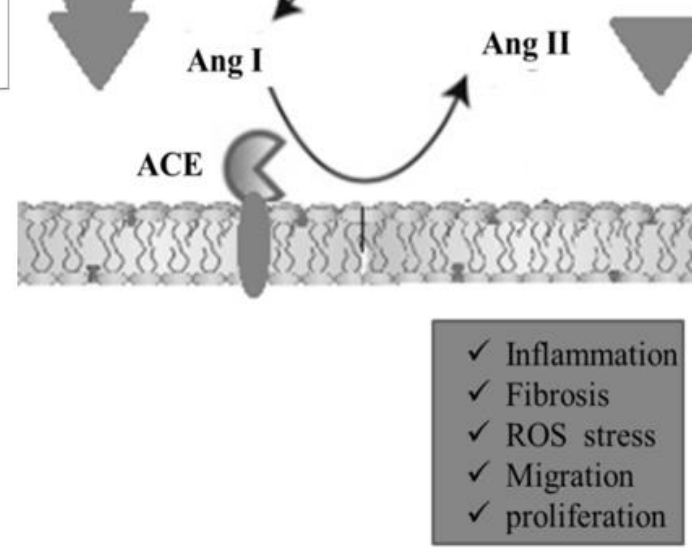

Renin

Renin

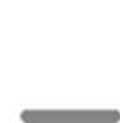

AT1 receptor

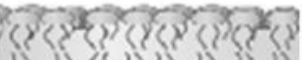

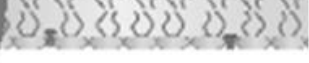

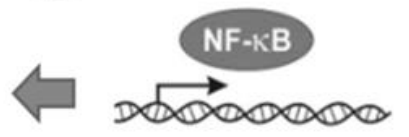

$\checkmark$ Migration

Figure 6: Valsartan decrease inflammatory cytokine expression and angiogenesis. Reduction of IFN$\gamma(\mathrm{A})$, TGF- $\beta(\mathrm{B})$, TNF- $\alpha(\mathrm{C})$, IL-6 (D), with valsartan $\left({ }^{* *} \mathrm{P}<0.01\right.$ and ${ }^{* * *} \mathrm{P}<0.001$ compared to control group). (E) Drug response for VEGF, VEGF-R and IL-6; (F) Schematic representation summarizing the molecular mechanisms of anti-tumor activities of valsartan in colorectal cancer.

\section{Discussion}

To the best of our knowledge, we firstly demonstrated valsartan's antiproliferative behavior and its association with 5-FU in CRC. Our data indicated that targeting the angiotensin II type I pathway may inhibit tumor growth and ameliorate fibrosis and inflammation through transforming growth factor beta pathway in colorectal cancer. There widespread evidence indicating that AT-II may perform an vital role in many types of cancers by modulating adhesion, angiogenesis, tumor growth, migration, invasion, and proliferation [14]. It has been shown in the Osumi et al study that the use of ARBs was associated with longer overall survival (OS) and progression-free survival (PFS) in metastatic CRC patients undergoing first-line bevacizumab-based chemotherapy. This suggests that RAS suppression may inhibit tumor growth and enhance survival [15]. Conclusions The effect of ACEIs or ARBs on disease progression or survival in cancer patients has been proven [16]. There has been some evidence to indicate that the use of these inhibitors may be related to improvement in cancer outcomes [16]; however, to further investigate this relationship, broader epidemiological 
studies with adequate information on drug dosage, frequency, and length are needed. Additionally, Patients with advanced CRC with ACEI / ARB and $\beta$-Blockers have been shown to have increased survival and reduced tumor growth and hospitalization levels [17]. Several clinical trials have also shown that RASIs can have therapeutic effects in a wide variety of cancers [18]. The survival advantage is tumor and stage dependent and ranges from 3 months (advanced non-small cell lung cancer (NSCLC)) to more than 25 months (metastatic renal cell carcinoma (RCC)) in retrospective studies. Moreover, the RASI medication response can not only differ with the type of tumor but can also rely on characteristics of certain tumors, treatment of cancer, type and dosage of RASI. More specifically, RCC, hepatocellular carcinoma (HCC), pancreatic ductal adenocarcinoma (PDAC), glioblastoma, cancer of the urinary tract, and non-small cell lung cancer tend to relate to the groups of sensitive tumors, whereas breast cancer does not respond to RASI. There are several studies about the activity of RAS inhibitors in NSCLC, gastric cancer, and colorectal cancer who received platinumbased chemotherapy and those with anti-VEGF therapy RCC, HCC, and CRC (e.g., sunitinib) [18].

The AT1R activity mediated via AT II, has been shown in numerous malignant tissues [19]. With regard to the role of ATII in proliferation and migration of cell, it is suggested that certain stages of tumor genesis and tumor progression play a role [20,21]. Blockade with AT1R antagonist resulted in a decrease in tumor growth [16]. The main actions of ARBs are mediated by inhibiting AT1R, and they have an enhanced inhibitory effect on tumor growth [20]. However, some researchers have shown continuing tumor growth despite AT1R B treatment [21]. ARBs are widely used in clinical practice every day because of their proven good tolerability, low side effects profile and well-known effectiveness [22]. The differences in structures between ARBs including variations in distribution, lipid solubility, biotransformation, bioavailability, plasma half-life and elimination [23]. The finding of the present study was to elucidate that tumor growth-induced CRC was suppressed by treatment valsartan in mice.

The AT1Rs can induce several signaling pathways such as PI3K/Ras/mTOR/AKT which involved in regulation of metastasis, apoptosis and cellular proliferation [24, 25]. Recent studies reported that $\mathrm{PI} 3 \mathrm{~K} / \mathrm{mTOR} / \mathrm{AKT}$ pathway is responsible for human colorectal cancers [26, 27]. Jaclyn et al, have shown that captopril and irbesartan (as RAS blocker) decreased tumor growth in CRC liver metastases that correlated with the reduction of central microvascular density significantly [28]. Another study showed that treatment with candesartan as an AT1RB decreased tumor growth in an experimental breast cancer model [29]. According to our results, Miyajima et al. demonstrated that the administration of candesartan reduced the development of lung metastases in a murine model of metastatic renal cell carcinoma [30]. Irbesartan enhanced Kupffer cell anti-tumor activity and reduced the severity of liver metastasis in people with CRC $[28,31]$. Some studies have shown in vivo enhancement of tumor vascularization by ARBs and direct stimulation of AT2R [7, 32]. Cancer cell metastatic properties are determined by their migration and invasion. Therefore, a primary strategy in cancer treatment is impeding migration and cancer cell invasion that AT1RB such as valsartan is involved in this process [14]. Although other mechanisms may act synergistically in the development of CRC, in recent years it has been explained that the inflammation and oxidative stress indicators play an important part in this process [33]. Our finding investigated the anti-tumor mechanisms of valsartan in vivo and in vitro in a tumor xenograft model. We showed that valsartan elicited its antitumor properties by reducing tumor weight and size, inducing cell senescence, apoptosis, oxidative stress and inflammatory responses inhibiting cell cycle progression, in both CRC cells and mouse model CRC.

ROS play a vital role in apoptosis induction and diminish of cell viability in the early stage of cancer $[4,34]$. AT1RB have a critical role in inhibition of tumor growth by apoptosis induction, cellular proliferation and vascular invasion [35]. In prostate cancer cells, telmisartan inhibited proliferation but this effect was not observed with valsartan, losartan, irbesartan or candesartan [36]. However, a recent report in human prostate cancer cells indicated that losartan may increase apoptosis [35]. The affect cell viability $[37,38]$ or proliferation [7] did not show with candesartan. There is an impact on 
the invasive potential of tumor cells when they have treated with ARBs [39]. According to our results, demonstrated that valsartan increases cellular senescence by up-regulation of inflammatory mediators, inducing oxidative stress, increasing ROS generation in CRC cells

Moreover, our data showed that tumor-related NO can develop and suppress cancer growth depending on context [40]. It was the suggestion that AT2Rs are important in NO production [41]. Nguyen et al demonstrated that AT2R inhibition reduces iNOS levels while AT1R blockade was likely to increase iNOS levels due to a proportional rise in AT2R activity [40]. Taken together, our findings have shown that co-administration of valsartan and 5-FU increased oxidant marker and reduced antioxidant indicators and tumor proliferation CRC in this experimental model. This results showed that the shift in oxidant-antioxidant status could be one of the underlying mechanisms that led to valsartan action against CRC. Lung metastasis involves the invasion and movement of tumor cells into the lungs through the bloodstream [42]. Several studies have been reported that ATII to accelerate lung metastasis of cancer cells [43]. Recent evidence suggests that AT1R B, valsartan (40 $\mathrm{mg} / \mathrm{kg} /$ day), suppressed the effect of AT-II [44]. The results of this study indicate that valsartan improve lung metastasis of colorectal cancer via downregulation of inflammatory markers such as IL-6 and the level of VEGF and VEGFR-1. The present finding also supports Deshayes et al study which concluded that treatment administration of ATIIR1 blockers (ARBs) have been associated with a lower occurrence of metastases and lower VEGF levels [45]. The finding is consistent with the finding of Ager et al, which administration with candesartan and irbesartan restrained growth of tumor, angiogenesis, and metastasis in an animal model of CRC metastasis to the lung [2]. Based on the results, valsartan through induction of ROS can be obstructed lung metastasis. This is supported by Piskounova study which reveals that enhancement of oxidative stress in metastasis derive of human melanoma cells suppresses distant metastasis [46].

In conclusion, the current study indicated that valsartan inhibited CRC growth by inhibition of cell proliferation, apoptosis, migration, and enhancement pro-inflammatory responses. 


\section{Materials and Methods}

\subsection{Chemicals and drugs}

Valsartan was obtained from Cayman Co, Michigan which dissolved in ethanol and diluted in sterile water, while Fluorouracil (5-FU) was a gift from the Mashhad University of Medical Science and diluted in sterile water. The Netherlands Dulbecco's Modified Eagle Medium: Nutrient Mixture F-12 (DMEM/F12), fetal bovine serum (FBS), penicillin and streptomycin were purchased from Gibco BRL, Life Technologies Inc. (Gaithersburg, MD, USA).

\subsection{Cell culture}

The CT26 cell line was cultured in DMEM/F12 medium and subsequently incubated with $10 \%$ heatinactivated $\mathrm{FBS}$ and $1 \%$ streptomycin at $5 \% \mathrm{CO} 2$ at $37^{\circ} \mathrm{C}$. In its exponential log step, the CT26 cells were harvested using trypsin-EDTA.

\subsection{MTT assay}

The anti-cancer properties of valsartan, 5-FU, and the both of them on CRC cells were assessed using the MTT test before and after cell treatment within 24 hours as described previously [47].

\subsection{Apoptosis analyses}

Annexin-V-fluorescein isothiocyanate (FITC) and propidium iodide (PI) assay kits (Cayman chemical Co, Michigan ) were used to assess apoptotic cell death [48]. Briefly, the cells of CRC were distributed at a density of $2 \times 106$ cells per well in 6-well plates, and treated with Valsartan $(1 \mathrm{mM})$ for 24 hr. The cell extracts were suspended in $200 \mu \mathrm{L}$ 1X Annexin V binding buffer. After centrifuging cells (for 5 minutes at $400 \mathrm{~g}$ ), the cell pellets were re-suspended in Annexin V-FITC/PI staining solution and have been incubated at room temperature for 15 minutes. Subsequently, the rate of viable, secondary necrotic cells, early and late apoptotic were quantified using FACSCalibur flow cytometer (BD Biosciences-US) and FlowJo software [49].

\subsection{In vitro migration assays}

Valsartan 's ability to inhibit the migratory activity of CT-26 cells was investigated using an in vitro migration assay, as followed mentioned. At their $5 \times \mathrm{IC} 50 \mathrm{~s}$, the cells were exposed to the drugs. At the beginning of the exposure, images were taken with those taken after 6, 10, 22, 28 and 36 hours.

\subsection{In vivo model for $C R C$}

Twenty-four Inbred BALB/c mice (average old 7 weeks) were purchased from the Pasteur Institute (Tehran, Iran). They have been held in normal conditions approved by the Institute animal ethics committee (temperature $23 \pm 2{ }^{\circ} \mathrm{C}$, humidity of $53 \pm 3 \%$ and $12 \mathrm{~h}$ Period light / dark). Approximately two weeks after the tumor cells implant, when tumors reached a volume of $80-100 \mathrm{~mm} 3$ [50], mice were separated into four groups randomly as below: (1) Control (Untreated mice), (2) 5-FU (treated with $5 \mathrm{mg} / \mathrm{kg}$ every other day, intraperitoneally) [51], (3) Valsartan (treated with $40 \mathrm{mg} / \mathrm{kg} / \mathrm{day}$, orally) [52], (4) combination (treated with $5 \mathrm{mg} / \mathrm{kg} 5-\mathrm{FU}$ every other day i.p. and Valsartan $40 \mathrm{mg} / \mathrm{kg} / \mathrm{day}$, orally) (Fig 1). The size of tumor was assessed every other day with the digital caliper and the tumor volume was determined according to the formula: Tumor volume $=($ tumor length $) \times($ tumor width) $2 / 2$. At the end of the fourteen day, tumor tissue was harvested for assessment of histological and biochemical dimensions.

\subsection{In vivo model for developing lung metastasis}

Twenty-four Inbred BALB/c animals were separated into 4 groups ( $\mathrm{n}=6)$ : a control group (no treated), a 5-FU-treated group (5mg/kg every other day (ip)), a valsartan-treated group (40 mg/kg/day (oral gavage)), and a combination-treated group $(5 \mathrm{mg} / \mathrm{kg} 5$-FU every other day (ip) plus valsartan 40 
$\mathrm{mg} / \mathrm{kg} /$ day (oral gavage)). Lung metastases in the animals were produced by injecting $2 \times 10^{6} \mathrm{CT} 26$ cells in $100 \mathrm{~mL}$ of PBS intravenous via the lateral tail vein. Treatment was started 24 hours after injection and continued for 10 days (Fig 7) [53]. The mice were sacrificed at 11 days, and the lungs were separated. The lung weights were measured, and tissues fixed in Bouin's solution for counting tumor colonies.

\subsection{Histological evaluation}

The tumors (CRC model) and lung (CRC lung metastasis model) were isolated and then put in $10 \%$ formalin overnight. After dehydration, the tumors were coated in paraffin and chopped with a microtome. The sections were stained with Hematoxylin-Eosin (H\&E) and Masson's trichrome stains and studied with light microscopy ( $\times 40$ magnification).

\section{9. $q R T-P C R$}

RNAs was quantified in the CRC tissues after treatment with valsartan and 5-FU and complementary DNAs (cDNA) synthesized according to the manufacturers' protocol. The real-time PCR analysis was carry out using specific primers Macrogene (Macrogene Co, Seoul, Korea) by light cycler real-time Polymerase chain reaction (PCR) (Roche Diagnostics, Mannheim, Germany). Glyceraldehyde 3phosphate dehydrogenase (GAPDH) was used as a housekeeping control gene as described [54].

\subsection{Oxidant and anti-oxidant assessments}

The tumor and lung specimen were homogenized with a PBS (phosphate buffer solution with pH 7.4). The homogenates for 15 min were centrifuged and malondialdehyde (MDA), total thiol, superoxide dismutase (SOD) [44], catalase (CAT) [28] were measured.

\subsection{Malondialdehyde}

The biomarker of lipid peroxidation is MDA. The supernatant of tumor and lung homogenate was affixed to thiobarbituric acid. Then the absorbance was measured at 535nm wavelength using spectrophotometer against a blank and the MDA content was determined [13].

\subsection{Total thiol group}

Measurement of total thiol groups using dithionitrobenzoic acid (DTNB). In Tris-EDTA buffer ( $\mathrm{pH}=8.6$ ), the tumor and the lung supernatants were incubated with DTNB. The composition was then kept for ten min at room temperature, and the wavelength of the absorbance was $412 \mathrm{~nm}$. The total thiol content was calculated using below formula [55]. Total thiol concentration $(\mathrm{mM})=(\mathrm{A} 2-\mathrm{A} 1-\mathrm{B}) \times 1.07 /(0.05 \times 13.6)$

\subsection{Superoxide dismutase}

Measurement of SOD activity was based on the Pyrogallol auto-oxidation and suppression of conversion of MTT to formazan. The formazan was dissolved in DMSO and its absorption was read at a wavelength of $570 \mathrm{~nm}$ [56].

\subsection{Catalase}

The CAT behavior was calculated using spectrophotometer with a wavelength of $240 \mathrm{~nm}$ based on the decomposition of hydrogen peroxide (H2O2) [57].

\subsection{Reactive oxygen species (ROS) evaluation by DCFH-DA method}

For evaluation of intracellular ROS production used from the change of redox-sensitive dye 2', $7^{\prime}$ dichlorodihydrofluorescein diacetate (DCFH-DA) to a green fluorescent product, dichlorofluorescein 
(DCF). Briefly, the CT-26 cells were treated with valsartan, 5-FU, and the combination of valsartan and 5-FU for 6 hours, in complete medium. Then the cells were incubated in fresh culture medium plus DCFH-DA for $30 \mathrm{~min}$. After washing Per PBS three times, the fluorescence values were measured using a fluorimeter and cells were imaged with an inverted fluorescence microscope [34].

\subsection{Nitric oxide (NO) metabolites}

The tumor NO metabolites were determined using the Griess reaction using colorimetric assay (Promega Corp. USA) according to the standard protocol [58].

Evaluation of Interleukin-6 (IL-6), Interferon gamma) IFN- $\gamma$ (, TGF- $\beta$, tumor necrosis factor alpha (TNF$\alpha)$ and angiogenic markers

Quantification of IL-6, IFN- $\gamma$, TGF- $\beta$, and TNF- $\alpha$ in colon cancer cells was performed using cytokine detection ELISA kits (eBioscience). Also the level of VEGF, and VEGF receptor-1 (VEGFR1) was measured using ELISA kits (Zellbio) according to the manufacturers' protocol.

4.17. In silico and heat map analysis of valsartan response signature

The Interaction of valsartan with IL- $\beta$, TNF $\alpha$, MCP-1, AT1R, TGF- $\beta$, oxidant/antioxidant factors, IL-6, and VEGF/VEGFR1 markers was evaluated by autodock and LigPlot+ software or by R software.

\subsection{Statistical analysis}

Data are presented as mean \pm SEM and analyzed by One-way ANOVA followed by post LSD comparison tests. Data analysis was undertaken using SPSS v.20 statistical software (IBM, Chicago). Differences for a $\mathrm{P}<0.05$ were considered statistical significant.

Author Contributions: methodology, Fereshteh Asgharzadeh, Asma Mostafapour, Forouzan Amerizadeh, Farzad Rahmani, Reihaneh Sabbaghzadeh, Maryam Fakhraei, Alieh Farshbaf, Majid Khazaei.; software, Fereshteh Asgharzadeh.; formal analysis, Fereshteh Asgharzadeh, Asma Mostafapour, Forouzan Amerizadeh, Farzad Rahmani, Reihaneh Sabbaghzadeh,.; writing - original draft preparation, Fereshteh Asgharzadeh, Asma Mostafapour, Forouzan Amerizadeh, Farzad Rahmani.; writing-review and editing, Fereshteh Asgharzadeh, Amir Avan, Farzad Rahmani, Reihaneh Sabbaghzadeh, Seyed Mahdi Hassanian, Gordon A Ferns, William C. Cho, Majid Khazaei.; supervision, Seyed Mahdi Hassanian, Amir Avan Majid Khazaei.; funding acquisition, Majid Khazaei. All authors have read and agreed to the published version of the manuscript.",

Funding: This research was partly supported by National Institute for Medical Research Development, grant No. 971176 and Mashhad University of Medical Sciences, grant No. 971798 (Amir Avan, Majid Khazaei).

Acknowledgments: The authors would like to thank the Mashhad University of Medical Sciences for supporting this study.

Conflicts of Interest: The authors declare no conflict of interest. 


\section{References}

1. 1. Navarro, M.; Nicolas, A.; Ferrandez, A.; Lanas, A., Colorectal cancer population screening programs worldwide in 2016: An update. World journal of gastroenterology 2017, 23, (20), 3632-3642.

2. 2. Ager, E. I.; Neo, J.; Christophi, C., The renin-angiotensin system and malignancy. Carcinogenesis 2008, 29, (9), 1675-1684.

3. 3. Deshayes, F.; Nahmias, C., Angiotensin receptors: a new role in cancer? Trends in Endocrinology E Metabolism 2005, 16, (7), 293-299.

4. 4. Eftekhari, A.; Ahmadian, E.; Panahi-Azar, V.; Hosseini, H.; Tabibiazar, M.; Maleki Dizaj, S., Hepatoprotective and free radical scavenging actions of quercetin nanoparticles on aflatoxin B1induced liver damage: in vitro/in vivo studies. Artificial cells, nanomedicine, and biotechnology 2018, 46, (2), 411-420.

5. 5. Mann, S. J.; Christos, P. J., ACE Inhibitor s and ARB s: Do They Reduce the Risk of Cancer? The Journal of Clinical Hypertension 2014, 16, (1), 6-7.

6. 6. Fujita, M.; Hayashi, I.; Yamashina, S.; Fukamizu, A.; Itoman, M.; Majima, M., Angiotensin type 1a receptor signaling-dependent induction of vascular endothelial growth factor in stroma is relevant to tumor-associated angiogenesis and tumor growth. Carcinogenesis 2005, 26, (2), 271-279.

7. $\quad$ 7. Kosaka, T.; Miyajima, A.; Takayama, E.; Kikuchi, E.; Nakashima, J.; Ohigashi, T.; Asano, T.; Sakamoto, M.; Okita, H.; Murai, M., Angiotensin II type 1 receptor antagonist as an angiogenic inhibitor in prostate cancer. The Prostate 2007, 67, (1), 41-49.

8. 8. Wang, L.; Cai, S.; Zhang, C.; He, Y.; Zhan, W.; Wu, H.; Peng, J., Effects of angiotensin converting enzyme inhibitors and angiotensin II receptor blockers on angiogenesis of gastric cancer in a nude mouse model. Zhonghua wei chang wai ke za zhi= Chinese journal of gastrointestinal surgery 2008, 11, (6), $565-568$.

9. 9. Wang, Q.; Zhao, W.; Wu, G., Valsartan inhibits NPC cell line CNE-2 proliferation and invasion and promotes its sensitivity to radiation. European Journal of Cancer Prevention 2009, 18, (6), 510-517.

10. 10. Chujo, D.; Yagi, K.; Asano, A.; Muramoto, H.; Sakai, S.; Ohnishi, A.; Shintaku-Kubota, M.; Mabuchi, H.; Yamagishi, M.; Kobayashi, J., Telmisartan treatment decreases visceral fat accumulation and improves serum levels of adiponectin and vascular inflammation markers in Japanese hypertensive patients. Hypertension Research 2007, 30, (12), 1205-1210.

11. 11. Nishimura, T.; Hashimoto, J.; Ohkubo, T.; Kikuya, M.; Metoki, H.; Asayama, K.; Totsune, K.; Imai, Y., Efficacy and duration of action of the four selective angiotensin II subtype 1 receptor blockers, losartan, candesartan, valsartan and telmisartan, in patients with essential hypertension determined by home blood pressure measurements. Clinical and Experimental Hypertension 2005, 27, (6), 477-489.

12. 12. Yano, Y.; Hoshide, S.; Ishikawa, J.; Noguchi, C.; Tukui, D.; Takanori, H.; Tada, M.; Kanemaru, Y.; Yano, A.; Ishikawa, S., The differential effects of angiotensin II type 1 receptor blockers on microalbuminuria in relation to low-grade inflammation in metabolic hypertensive patients. American journal of hypertension 2007, 20, (5), 565-572.

13. 13. Collaboration, A. T., Effects of telmisartan, irbesartan, valsartan, candesartan, and losartan on cancers in 15 trials enrolling 138769 individuals. Journal of hypertension 2011, 29, (4), 623-635.

14. 14. Ishikane, S.; Takahashi-Yanaga, F., The role of angiotensin II in cancer metastasis: Potential of renin-angiotensin system blockade as a treatment for cancer metastasis. Biochem Pharmacol 2018, 151, 96-103.

15. 15. Osumi, H.; Matsusaka, S.; Wakatsuki, T.; Suenaga, M.; Shinozaki, E.; Mizunuma, N., Angiotensin II type-1 receptor blockers enhance the effects of bevacizumab-based chemotherapy in metastatic colorectal cancer patients. Molecular and clinical oncology 2015, 3, (6), 1295-1300.

16. 16. Mc Menamin, U. C.; Murray, L. J.; Cantwell, M. M.; Hughes, C. M., Angiotensin-converting enzyme inhibitors and angiotensin receptor blockers in cancer progression and survival: a systematic review. Cancer Causes \& Control 2012, 23, (2), 221-230.

17. 17. Engineer, D. R.; Burney, B. O.; Hayes, T. G.; Garcia, J. M., Exposure to ACEI/ARB and $\beta$-blockers is associated with improved survival and decreased tumor progression and hospitalizations in patients with advanced colon cancer. Translational oncology 2013, 6, (5), 539.

18. 18. Pinter, M.; Jain, R. K., Targeting the renin-angiotensin system to improve cancer treatment: Implications for immunotherapy. Sci Transl Med 2017, 9, (410).

19. 19. Childers, W. K., Interactions of the renin-angiotensin system in colorectal cancer and metastasis. Int J Colorectal Dis 2015, 30, (6), 749-52.

20. 20. Olin, J. L.; Veverka, A.; Nuzum, D. S., Risk of cancer associated with the use of angiotensin IIreceptor blockers. American Journal of Health-System Pharmacy 2011, 68, (22), 2139-2146. 
21. 21. Willis, L. M.; El-Remessy, A. B.; Somanath, P. R.; Deremer, D. L.; Fagan, S. C., Angiotensin receptor blockers and angiogenesis: clinical and experimental evidence. Clinical Science 2011, 120, (8), 307-319.

22. 22. Corrao, G.; Zambon, A.; Parodi, A.; Poluzzi, E.; Baldi, I.; Merlino, L.; Cesana, G.; Mancia, G., Discontinuation of and changes in drug therapy for hypertension among newly-treated patients: a population-based study in Italy. Journal of hypertension 2008, 26, (4), 819-824.

23. 23. Burnier, M., Telmisartan: a different angiotensin II receptor blocker protecting a different population? Journal of International Medical Research 2009, 37, (6), 1662-1679.

24. 24. Baldus, S. E.; Schaefer, K.-L.; Engers, R.; Hartleb, D.; Stoecklein, N. H.; Gabbert, H. E., Prevalence and heterogeneity of KRAS, BRAF, and PIK3CA mutations in primary colorectal adenocarcinomas and their corresponding metastases. Clinical Cancer Research 2010, 16, (3), 790-799.

25. 25. Du, N.; Feng, J.; Hu, L.-J.; Sun, X.; Sun, H.-B.; Zhao, Y.; Yang, Y.-P.; Ren, H., Angiotensin II receptor type 1 blockers suppress the cell proliferation effects of angiotensin II in breast cancer cells by inhibiting AT1R signaling. Oncology reports 2012, 27, (6), 1893-1903.

26. 26. Arqués, O.; Chicote, I.; Puig, I.; Tenbaum, S. P.; Argilés, G.; Dienstmann, R.; Fernández, N.; Caratù, G.; Matito, J.; Silberschmidt, D., Tankyrase inhibition blocks Wnt/ $\beta$-catenin pathway and reverts resistance to $\mathrm{PI} 3 \mathrm{~K}$ and $\mathrm{AKT}$ inhibitors in the treatment of colorectal cancer. Clinical Cancer Research 2016, 22, (3), 644-656.

27. 27. Rahmani, F.; Avan, A.; Hashemy, S. I.; Hassanian, S. M., Role of Wnt/ $\beta$ - catenin signaling regulatory microRNAs in the pathogenesis of colorectal cancer. Journal of cellular physiology 2018, 233, (2), 811-817.

28. 28. Neo, J. H.; Malcontenti - Wilson, C.; Muralidharan, V.; Christophi, C., Effect of ACE inhibitors and angiotensin II receptor antagonists in a mouse model of colorectal cancer liver metastases. Journal of gastroenterology and hepatology 2007, 22, (4), 577-584.

29. 29. Chen, X.; Meng, Q.; Zhao, Y.; Liu, M.; Li, D.; Yang, Y.; Sun, L.; Sui, G.; Cai, L.; Dong, X., Angiotensin II type 1 receptor antagonists inhibit cell proliferation and angiogenesis in breast cancer. Cancer letters 2013, 328, (2), 318-324.

30. 30. Miyajima, A.; Kosaka, T.; Asano, T.; Asano, T.; Seta, K.; Kawai, T.; Hayakawa, M., Angiotensin II type I antagonist prevents pulmonary metastasis of murine renal cancer by inhibiting tumor angiogenesis. Cancer Research 2002, 62, (15), 4176-4179.

31. 31. Wen, S. W.; Ager, E. I.; Neo, J.; Christophi, C., The renin angiotensin system regulates Kupffer cells in colorectal liver metastases. Cancer biology \& therapy 2013, 14, (8), 720-727. 32. Uemura, H.; Nakaigawa, N.; Ishiguro, H.; Kubota, Y., Antiproliferative efficacy of angiotensin II receptor blockers in prostate cancer. Current cancer drug targets 2005, 5, (5), 307-323.

33. 33. Yisireyili, M.; Uchida, Y.; Yamamoto, K.; Nakayama, T.; Cheng, X. W.; Matsushita, T.; Nakamura, S.; Murohara, T.; Takeshita, K., Angiotensin receptor blocker irbesartan reduces stress-induced intestinal inflammation via AT1a signaling and ACE2-dependent mechanism in mice. Brain, behavior, and immunity 2018, 69, 167-179.

34. 34. Negrei, C.; Hudita, A.; Ginghina, O.; Galateanu, B.; Voicu, S. N.; Stan, M.; Costache, M.; Fenga, C.; Drakoulis, N.; Tsatsakis, A. M., Colon Cancer Cells Gene Expression Signature As Response to 5Fluorouracil, Oxaliplatin, and Folinic Acid Treatment. Frontiers in pharmacology 2016, 7, 172.

35. 35. Gong, Q.; Davis, M.; Chipitsyna, G.; Yeo, C. J.; Arafat, H. A., Blocking angiotensin II type 1 receptor triggers apoptotic cell death in human pancreatic cancer cells. Pancreas 2010, 39, (5), 581-594.

36. 36. Funao, K.; Matsuyama, M.; Kawahito, Y.; Sano, H.; Chargui, J.; Touraine, J.-L.; Nakatani, T.; Yoshimura, R., Telmisartan is a potent target for prevention and treatment in human prostate cancer. Oncology reports 2008, 20, (2), 295-300.

37. 37. Imai, N.; Hashimoto, T.; Kihara, M.; Yoshida, S.-i.; Kawana, I.; Yazawa, T.; Kitamura, H.; Umemura, S., Roles for host and tumor angiotensin II type 1 receptor in tumor growth and tumorassociated angiogenesis. Laboratory investigation 2007, 87, (2), 189.

38. 38. Kosugi, M.; Miyajima, A.; Kikuchi, E.; Kosaka, T.; Horiguchi, Y.; Murai, M., Effect of angiotensin II type 1 receptor antagonist on tumor growth and angiogenesis in a xenograft model of human bladder cancer. Human cell 2007, 20, (1), 1-9.

39. 39. Attoub, S.; Gaben, A. M.; Al - Salam, S.; Al Sultan, M.; John, A.; Nicholls, M. G.; Mester, J.; Petroianu, G., Captopril as a potential inhibitor of lung tumor growth and metastasis. Annals of the New York Academy of Sciences 2008, 1138, (1), 65-72.

40. 40. Nguyen, L.; Ager, E. I.; Neo, J.; Christophi, C., Regulation of colorectal cancer cell epithelial to mesenchymal transition by the renin angiotensin system. Journal of gastroenterology and hepatology 2016, $31,(10), 1773-1782$ 
41. 41. Fukumura, D.; Kashiwagi, S.; Jain, R. K., The role of nitric oxide in tumour progression. Nature Reviews Cancer 2006, 6, (7), 521.

42. 42. Maru, Y., The lung metastatic niche. J Mol Med 2015, 93, (11), 1185-92.

43. 43. Rodrigues-Ferreira, S.; Abdelkarim, M.; Dillenburg-Pilla, P.; Luissint, A. C.; di-Tommaso, A.; Deshayes, F.; Pontes, C. L.; Molina, A.; Cagnard, N.; Letourneur, F.; Morel, M.; Reis, R. I.; Casarini, D. E.; Terris, B.; Couraud, P. O.; Costa-Neto, C. M.; Di Benedetto, M.; Nahmias, C., Angiotensin II facilitates breast cancer cell migration and metastasis. PLoS One 2012, 7, (4), 20.

44. 44. Ishikane, S.; Hosoda, H.; Nojiri, T.; Tokudome, T.; Mizutani, T.; Miura, K.; Akitake, Y.; Kimura, T.; Imamichi, Y.; Kawabe, S.; Toyohira, Y.; Yanagihara, N.; Takahashi-Yanaga, F.; Miyazato, M.; Miyamoto, K.; Kangawa, K., Angiotensin II promotes pulmonary metastasis of melanoma through the activation of adhesion molecules in vascular endothelial cells. Biochem Pharmacol 2018, 154, 136-147.

45. 45. Deshayes, F.; Nahmias, C., Angiotensin receptors: a new role in cancer? Trends Endocrinol Metab 2005, 16, (7), 293-9.

46. 46. Piskounova, E.; Agathocleous, M.; Murphy, M. M.; Hu, Z.; Huddlestun, S. E.; Zhao, Z.; Leitch, A. M.; Johnson, T. M.; DeBerardinis, R. J.; Morrison, S. J., Oxidative stress inhibits distant metastasis by human melanoma cells. Nature 2015, 527, (7577), 186.

47. 47. Amerizadeh, F.; Rezaei, N.; Rahmani, F.; Hassanian, S. M.; Moradi-Marjaneh, R.; Fiuji, H.; Boroumand, N.; Nosrati-Tirkani, A.; Ghayour-Mobarhan, M.; Ferns, G. A.; Khazaei, M.; Avan, A., Crocin synergistically enhances the antiproliferative activity of 5-flurouracil through Wnt/PI3K pathway in a mouse model of colitis-associated colorectal cancer. Journal of cellular biochemistry 2018.

48. 48. Giovannetti, E.; Wang, Q.; Avan, A.; Funel, N.; Lagerweij, T.; Lee, J. H.; Caretti, V.; van der Velde, A.; Boggi, U.; Wang, Y.; Vasile, E.; Peters, G. J.; Wurdinger, T.; Giaccone, G., Role of CYB5A in pancreatic cancer prognosis and autophagy modulation. Journal of the National Cancer Institute 2014, 106, (1), djt346.

49. 49. Marjaneh, R. M.; Rahmani, F.; Hassanian, S. M.; Rezaei, N.; Hashemzehi, M.; Bahrami, A.; Ariakia, F.; Fiuji, H.; Sahebkar, A.; Avan, A.; Khazaei, M., Phytosomal curcumin inhibits tumor growth in colitis-associated colorectal cancer. Journal of cellular physiology 2018.

50. 50. Zhang, J.; Wang, X.; Liu, T.; Liu, S.; Jing, X., Antitumor activity of electrospun polylactide nanofibers loaded with 5-fluorouracil and oxaliplatin against colorectal cancer. Drug delivery 2016, 23, (3), 784-790.

51. 51. Marjaneh, R. M.; Rahmani, F.; Hassanian, S. M.; Rezaei, N.; Hashemzehi, M.; Bahrami, A.; Ariakia, F.; Fiuji, H.; Sahebkar, A.; Avan, A., Phytosomal curcumin inhibits tumor growth in colitis associated colorectal cancer. Journal of cellular physiology 2018, 233, (10), 6785-6798.

52. 52. Liu, Y.-H.; Yang, X.-P.; Sharov, V. G.; Nass, O.; Sabbah, H. N.; Peterson, E.; Carretero, O. A., Effects of angiotensin-converting enzyme inhibitors and angiotensin II type 1 receptor antagonists in rats with heart failure. Role of kinins and angiotensin II type 2 receptors. The Journal of clinical investigation 1997, 99, (8), 1926-1935.

53. 53. Li, Y.; Wang, C.; Li, D.; Deng, P.; Shao, X.; Hu, J.; Liu, C.; Jie, H.; Lin, Y.; Li, Z.; Qian, X.; Zhang, H.; Zhao, Y., 1H-NMR-based metabolic profiling of a colorectal cancer CT-26 lung metastasis model in mice. Oncol Rep 2017, 38, (5), 3044-3054.

54. 54. Dinarvand, P.; Hassanian, S. M.; Weiler, H.; Rezaie, A. R., Intraperitoneal administration of activated protein C prevents postsurgical adhesion band formation. Blood 2015, 125, (8), 1339-48.

55. 55. Bargi, R.; Asgharzadeh, F.; Beheshti, F.; Hosseini, M.; Sadeghnia, H. R.; Khazaei, M., The effects of thymoquinone on hippocampal cytokine level, brain oxidative stress status and memory deficits induced by lipopolysaccharide in rats. Cytokine 2017, 96, 173-184.

56. 56. Elmi, S.; Sallam, N. A.; Rahman, M. M.; Teng, X.; Hunter, A. L.; Moien-Afshari, F.; Khazaei, M.; Granville, D. J.; Laher, I., Sulfaphenazole treatment restores endothelium-dependent vasodilation in diabetic mice. Vascular pharmacology 2008, 48, (1), 1-8.

57. 57. Aebi, H., [13] Catalase in vitro. Methods in enzymology 1984, 105, 121-126.

58. 58. Nematollahi, S.; Nematbakhsh, M.; Haghjooyjavanmard, S.; Khazaei, M.; Salehi, M., Inducible nitric oxide synthase modulates angiogenesis in ischemic hindlimb of rat. Biomed Pap Med Fac Univ Palacky Olomouc Czech Repub 2009, 153, (2), 125-9. 